\title{
Evaluation of transient thrombocytopenia in the postoperative period of aortic valve
} replacement.

\begin{abstract}
Henrique Figueiredo Prado*, Guilherme Machado de Figueiredo Murari, Carlos Fernando Ramos Lavagnoli, Elaine Soraya Barbosa de Oliveira, Karlos Alexandre de Sousa Vilarinho, Pedro Paulo Martins de Oliveira, Orlando Petrucci, LIndemberg da Mota Silveira Filho.
\end{abstract}

\begin{abstract}
Transient thrombocytopenia is common after surgery for aortic valve replacement (AVR), but rarely studied systematically. Severe thrombocytopenia (platelets $<50000 / \mathrm{mL}$ ) often requires replacement of platelets and other blood products and exposure to their risks. The aims of this study are to verify the occurrence of complications in patients presenting with severe post op thrombocytopenia (SPOT) after AVR and differences between biological (BP) and mechanical prostheses (MP) in relation to post-op thrombocytopenia. Methods: Retrospective study with 110 patients undergoing AVR surgery with cardiopulmonary bypass, at Hospital de Clínicas, State University of Campinas (Unicamp) between 2011 and 2017. Patients with previous cardiac surgery or other associated cardiac procedures were excluded. Demographic, pre, intra and post-operative data were analyzed. Results: Age and surgical risk EuroSCORE were higher in those who received BP. The total platelets number in the post-op was lower in BP than MP. There was no difference in bleeding and transfusion volumes between patients with BP and MP. Considering all patients, those with SPOT presented lower hemoglobin rates and higher blood transfusion volume, but there was no difference in bleeding volumes. Conclusions: SPOT in AVR was more prevalent in patients who received BP. Patients with SPOT did not present more bleeding volumes but received higher blood transfusion than patients without SPOT.
\end{abstract}

Key words: transient trombocytopenia, aortic valve replacement, cardiac surgery.

\section{Introduction}

Transient thrombocytopenia is a common phenomenon after AVR, however, it has rarely been studied systematically[1]. Postoperative transient thrombocytopenia is commonly observed in cardiac surgeries with the use of cardiopulmonary bypass. Severe thrombocytopenia (ST), is defined as lower platelet count $50 \times 10 \% / \mathrm{uL}$, and when present in the postoperative, it is considered a potential adverse outcome due to the risk of bleeding or clotting disorders ${ }^{[2]}$. The maintenance of ST in the days following the procedure often requires intravenous replacement of platelets, anti-fibrinolytic agents use and may be associated with a greater need for replacement of other blood products and exposure to their risks. The aims of this study are to verify the occurrence of complications in patients presenting with severe post-op thrombocytopenia (SPOT) after AVR and differences between biological (BP) and mechanical prostheses (MP) in relation to postoperative thrombocytopenia.

\section{Results and Discussion}

Retrospective study with a 110 patients undergoing AVR with cardiopulmonary bypass, at Hospital de Clínicas, State University of Campinas (Unicamp) between 2011 and 2017. Patients with previous cardiac surgery or other associated cardiac procedures were excluded. We observed $5.45 \%$ hospital death and $3.65 \%$ bleeding reoperations. Seventy-two patients (65.5\%) received BPs and the latter had age and EuroSCORE higher than those who received MP. The rate of platelets in the postop was lower among BP than MP (146+/-40 vs. $76+/-46$ thousand, $p<0.001)$. There was no difference in bleeding and transfusion volume. Of the total, those with SPOT presented lower hemoglobin rates and higher blood transfusion volume.
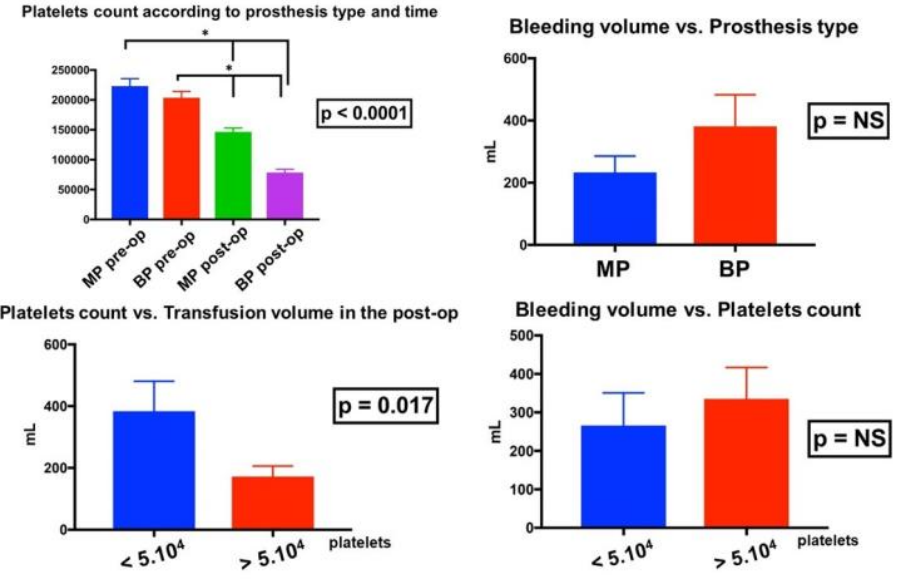

Image 1. Upper level: Platelets count and bleeding volume according to prosthesis. Lower: Transfusion and bleeding volumes in patients with SPOT vs no SPOT

\section{Conclusions}

SPOT in AVR was more prevalent in patients who received BP. Patients with SPOT did not present more bleeding volumes but received higher blood transfusion than patients without ST.

\section{Acknowledgement}

We would like to thank all those involved in the development of the project and the CNPq for their investment in teaching and research.

\footnotetext{
1 Sun X, Hill PC, Ellis J, Corso PJ, Taylor-Panek SL, Chen F. Ten-year screening for thrombocytopenia after aortic valve replacement. Interactive CardioVascular and Thoracic Surgery 18. 2014, 562-567.

${ }^{2}$ Ferraris VA, Brown JR, Despotis GJ, Hammon JW, Reece TB, Saha SP, et al Society of Thoracic Surgeons Blood Conservation Guideline Task Force. 2011. Ann Thorac Surg. 2011, 91:944-82.
} 